\title{
Can nocturnal emergency surgery be reduced?
}

\author{
D J SHERLOCK, J RANDLE, M PLAYFORTH, R COX, R T J HOLL-ALLEN
}

\begin{abstract}
A prospective study of emergency operations was performed over three months in a district general hospital. Before starting surgery surgeons completed a questionnaire recording clinical details together with time of admission and were asked to state whether in their opinion the case could be safely deferred until the next morning assuming operating time was available. Of 251 operations performed, forms were completed for 244. According to the surgeons, 62 could have been safely deferred, and subsequent independent analysis indicated a further 24 could also have been safely postponed, giving a total of 86 cases $(35 \%)$. Of the remainder, 71 were started between 2300 and 0900 , and of these $51(71 \%)$ were major operations requiring an experienced surgeon. The results suggest that, although the volume of nocturnal major surgery could not be reduced, the introduction of a daily emergency theatre session for cases seen during the day and those which could be deferred from the previous night would appreciably reduce the overall workload of nocturnal surgery at present done by the resident junior staff.
\end{abstract}

This study will have important implications if, as has been suggested, the number of junior medical staff on call at night for emergencies is reduced.

\section{Introduction}

In the United Kingdom most emergency duties, including operations, are performed by doctors in training. Although most nocturnal operations are undertaken for compelling clinical reasons, some appear to be done because it is impossible or inconvenient to defer them until the next morning. The proportion of operations that could be deferred will vary between specialties as well as hospitals. Until now this subject appears to have received little attention, but, if proposals to reduce the number of junior staff are implemented, it is during "out of hours" surgery that repercussions will occur.

Although the effects of fatigue on performance are now recognised, surgeons are still expected to operate for long periods at night without a critical study being made of the necessity for

\footnotetext{
Department of Surgery, East Birmingham Hospital, Birmingham B9 5ST

D J SHERLOCK, FRCS, surgical registrar

J RANDLE, FRCS, surgical registrar

M PLAYFORTH, FRCS, surgical registrar

R COX, FRCS, surgical registrar

R T J HOLL-ALLEN, MD, FRCs, consultant surgeon

Correspondence to: Mr R T J Holl-Allen.
}

this. Clearly, if a case can be deferred with safety both patient and surgeon will benefit if surgery is not carried out by a relatively tired surgeon. Operations should not be delayed until late at night, except for compelling clinical reasons.

A prospective study of emergency operations done in a large district general hospital over three months was set up to assess the number undertaken, the indications for the operations, the timing of the operations, and what proportion, if any, could have been deferred with safety until a hypothetical 0900 list the next morning. At the same time we determined whether any changes in practice could reduce the nocturnal workload.

\section{Patients and methods}

This hospital is a large district general hospital serving a population of roughly 300000 and admitting emergency cases every day. In common with many hospitals, it does not have a separate theatre available for emergency operations while elective surgery is being performed during the day. All emergency general surgical operations in adults and children undertaken from June to August 1983 were included in this study. Orthopaedic, otolaryngological, and thoracic cases and cases of non-abdominal trauma, although admitted to the hospital, were excluded. An emergency operation was defined for the purposes of the study as one in which the degree of urgency, as assessed by the admitting surgical team, was such that postponement until the next elective list was not possible. All cases were submitted to surgery as soon as circumstances permitted, in the normal manner.

Surgeons performing emergency operations were asked to complete a questionnaire before the operation began. As well as recording the provisional diagnosis and indications for surgery, details of whether the operation could be deferred safely until 0900 the next or same morning were included. The time the operation was started together with any reasons for delay were noted. Finally, the postoperative findings were recorded, and the surgeon gave a second opinion of whether the patient would have suffered any adverse effects had surgery been deferred. At all times the grades of the surgeons operating and making the decisions were noted, and all data were audited independently.

\section{Results}

Of 810 patients admitted during the study period, 251 underwent emergency surgery. Of these 251 , full details were available for 244 $(97 \%)$ and form the subject of this analysis. Most operations were done by junior surgical staff, registrars undertaking $185(76 \%)$, senior house officers $46(19 \%)$, house officers four $(1.7 \%)$, and consultants eight $(3.3 \%)$. Timing of the operations showed that $44(18 \%)$ were done between 0900 and 1700 , using time available between and after elective surgery. Just over half (137) were started between 2000 and 0200 ; few were started thereafter.

Initially, 62 cases were assessed by the operating surgeon to be suitable for the 0900 list. After review, however, 24 cases appeared to have been incorrectly categorised by the more junior surgeons taking part in the study. The 24 cases comprised 14 abscesses and 10 minor anal conditions and, when added to the initial 62 deferrable cases, made a total of $86(35 \%)$ that could have safely been deferred to a 0900 list 
the next morning (table I). Thirty eight $(35 \%)$ of the operations started between 2000 and 2400 could have been deferred until the next morning: in other words, 38 patients with deferrable conditions underwent surgery during the hours when non-deferrable cases had priority. Furthermore, in none of these cases was it considered to be likely that postponement of surgery would lead to any complications. Subtraction of these cases from the total workload during that period indicated that deferrable cases occupied a large proportion of the nocturnal operation time despite their minor nature. In addition, they were often delayed until the more serious cases had been treated; the mean (range) time for delay was $5 \cdot 2(0-16)$ hours.

TABLE I-Details of cases that could have been deferred

\begin{tabular}{lc}
\hline Condition requiring surgery & $\begin{array}{c}\text { No of } \\
\text { patients }\end{array}$ \\
\hline Assorted abscesses & 23 \\
Minor anal conditions & 26 \\
Pain in right iliac fossa (cause uncertain) & $18^{*}$ \\
Hernia & 2 \\
Assorted intra-abdominal conditions & 13 \\
Gastrointestinal conditions & $1 \dagger$ \\
Amputation & 2 \\
Lost foreign body & 1 \\
\hline Total & 86 \\
\hline
\end{tabular}

* Twelve of these proved to have a normal appendix. †Perforated duodenal ulcer.

Further analysis was facilitated by subdividing the cases that could not be deferred into two groups according to whether surgery was major or minor (table II). Surgery classified as minor included relatively straightforward and less technically demanding operations; surgery classified as major required a more experienced surgeon of registrar or consultant grade. Separating the cases in this manner showed that $71(45 \%)$ of the 158 patients with non-deferrable conditions underwent a major procedure requiring an experienced surgeon. Taking 2300 as an arbitrary time after which surgery is least acceptable, 71 undeferrable operations were performed between 2300 and 0900 , of which 51 were major.

\begin{tabular}{|c|c|}
\hline Condition requiring surgery & $\begin{array}{c}\text { No of } \\
\text { patients }\end{array}$ \\
\hline \multicolumn{2}{|l|}{ Major } \\
\hline $\begin{array}{l}\text { Upper gastrointestinal tract condition } \\
\text { Small and large intestinal conditions } \\
\text { Vascular conditions } \\
\text { Biliary conditions } \\
\text { Burst wounds } \\
\text { Hernias requiring small bowel resection }\end{array}$ & $\begin{array}{c}20^{*} \\
26 \\
8 \\
5 \\
3 \\
9\end{array}$ \\
\hline Total & 71 \\
\hline $\begin{array}{l}\text { Appendicectomy } \\
\text { Hernia } \\
\text { Circumcision } \\
\text { Scrotal exploration }\end{array}$ & $\begin{array}{r}74 \\
5 \\
2 \\
6\end{array}$ \\
\hline Total & 87 \\
\hline
\end{tabular}

Half the patients with conditions that could not be deferred experienced delay in undergoing surgery, the mean (range) delay being $3 \cdot 8(0-13)$ hours. Furthermore, in 34 of the 71 operations done between 2300 and 0900 delay of some nature occurred, the mean delay being 6.0 hours; the remainder were treated promptly. The predominant reasons for delay were unavailability of theatre space, theatre staff, or anaesthetic services owing to use by other specialties.

\section{Discussion}

We examined the volume and timing of emergency general surgical operations over three months in this hospital. At the same time, a simple audit assessed which patients could have been left for operation until the next morning and which required immediate surgery. The results confirm that a considerable amount of surgery is done at night, 137 operations (57\%) being begun between 2000 and 0200 and 51 major operations $(21 \%)$ after 2300 . At the same time they confirm that there was little abuse of the system or unnecessary surgery performed. If, however, an emergency theatre session had been available with adequate support staff the next morning $86(35 \%)$ fewer operations need have been done during the night. Another benefit would have been some saving on overnight admissions as most of the cases were of a relatively simple nature.

As in most hospitals, the operations were done by surgeons who had already done a full day's work and were expected to do the same the next day. Although no data have been found concerning the effects of fatigue on surgical performance, surgeons are unlikely to be immune to the consequences of lack of sleep experienced by others. As well as easing the nocturnal workload, the introduction of a 0900 list would ensure that cases were undertaken by a fresh surgical team with the full support of facilities not usually available at night; indeed, a different decision might possibly be made by the next morning by a person not physically or mentally tired. The need for experience in preoperative decision making was also apparent in the study; the more experienced the surgeon, the greater his confidence that cases could be deferred until the next morning. Certainly, of the non-deferrable cases, $46(29 \%)$ needed surgery performed by an experienced registrar or consultant; $24(52 \%)$ of these operations occurred after 2300 .

The survey also showed that after the decision to operate had been made surgery was often delayed, the less urgent cases waiting until the more urgent had been dealt with (a mean of $5 \cdot 2$ compared with 3.8 hours). As noted, most delays were due to a lack of theatre time or staff; there was no evidence that these delays had any detrimental effect on any patient's physical well being. Delays could be reduced by improving facilities, including providing extra theatre space where necessary, and by providing adequate numbers of experienced surgical and support staff. An on call system for nursing staff would allow staffing levels to fluctuate in accordance with demand and simultaneously allow theatres to function as necessary.

Our results are relevant to the changes in medical practice and staffing that have recently been proposed. A reduction in the training grades would effectively reduce cover by registrars for out of hours emergencies if normal daily duties continued to be undertaken. As most emergency surgery is done by this grade at present, important alterations in current practice would be inevitable. Presumably consultants would be expected to deal with the increased workload. A natural reluctance to operate at night would lead to the postponement of less urgent cases as the obvious option, although an appreciable number of major operations would remain that could not be delayed in this way. To maintain the standard of the service other measures would inevitably be required to cope with this workload and avoid fatigue the next day in surgeons performing these duties in the absence of the present registrar grade.

If the amount of surgery done at night is reduced or suggested changes in medical staffing are eventually introduced, the provision of a daily emergency theatre session would reduce the workload at night, and this would be further reduced by a reallocation of resources within the hospital. An appreciable volume of major surgery would, however, still remain, requiring the attention of an experienced surgeon.

Further study of the problems posed by emergency surgery is required over a longer period and on a regional basis. Without an accurate knowledge of the extent of the problem the profession cannot hope to provide a working solution to maintain the standard of emergency surgical services and further government intervention would be inevitable.

We thank the consultant surgeons at this hospital, who permitted study of their patients, and other members of staff for their cooperation. We also thank P Sherlock for help preparing the manuscript. 